\title{
Hadley Cell Dynamics in a Virtually Dry Snowball Earth Atmosphere
}

\author{
Aiko VoigT \\ Max Planck Institute for Meteorology, and International Max Planck Research School on Earth System Modelling, \\ Hamburg, Germany
}

ISAAC M. HELD

Geophysical Fluid Dynamics Laboratory, Princeton, New Jersey

JOCHEM MAROTZKE

Max Planck Institute for Meteorology, Hamburg, Germany

(Manuscript received 17 March 2011, in final form 22 June 2011)

\begin{abstract}
The Hadley cell of a virtually dry snowball Earth atmosphere under equinox insolation is studied in a comprehensive atmospheric general circulation model. In contrast to the Hadley cell of modern Earth, momentum transport by dry convection, which is modeled as vertical diffusion of momentum, is important in the upper branch of the snowball Earth Hadley cell. In the zonal momentum balance, mean meridional advection of mean absolute vorticity is not only balanced by eddies but also by vertical diffusion of zonal momentum. Vertical diffusion also contributes to the meridional momentum balance by decelerating the Hadley cell through downgradient mixing of meridional momentum between its upper and lower branches. When vertical diffusion of momentum is suppressed in the upper branch, the Hadley cell strengthens by a factor of about 2. This is in line with the effect of vertical diffusion in the meridional momentum balance but in contrast with its effect in the zonal momentum balance. Neither axisymmetric Hadley cell theories based on angular momentum conservation nor eddy-permitting Hadley cell theories that neglect vertical diffusion of momentum are applicable to the snowball Earth Hadley cell. Because the snowball Earth Hadley cell is a particular realization of a dry Hadley cell, these results show that an appropriate description of dry Hadley cells should take into account vertical transport of momentum by dry convection.
\end{abstract}

\section{Introduction}

On Earth as well as other rotating planets such as Mars and Venus, the zonal-mean circulation of the tropical atmosphere is dominated by the Hadley cell. Motivated by this, the development of Hadley cell theories that capture the relation of the Hadley cell to internal parameters such as the tropopause height and the pole-toequator temperature contrast and its dependence on external parameters such as the rotation rate and the solar constant has for a long time been a vivid topic of atmospheric dynamics (e.g., Lorenz 1967; Schneider 2006). While geophysical fluid dynamics provides the underlying

Corresponding author address: Aiko Voigt, Max Planck Institute for Meteorology (MPI-M), Bundesstrasse 53, Hamburg D-20146, Germany.

E-mail: aiko.voigt@zmaw.de equations, the challenge in constructing Hadley cell theories is to identify which terms of these equations govern the Hadley cell under investigation, and which terms can be neglected.

The long-term goal is a complete Hadley cell theory that is applicable to a wide range of climates and planetary atmospheres and incorporates the effect of latent heat release. It is sometimes stated that an important step toward this theory is the development of a Hadley cell theory for dry atmospheres (Schneider 2006). Since there is no condensable component and hence no latent heat release in dry atmospheres, one might also assume that dry Hadley cells are easier to understand than moist Hadley cells. As a consequence, dry Hadley cells have been studied in analytical models and idealized atmospheric general circulation models (AGCMs) as described below. However, dry Hadley cells so far have not been investigated in a comprehensive AGCM, in 
contrast to moist Hadley cells (Satoh et al. 1995; Williams 1988a,b). ${ }^{1}$

This gap motivates us to examine the Hadley cell dynamics of a snowball Earth atmosphere in a comprehensive AGCM. The snowball Earth atmosphere is a particular realization of a dry atmosphere due to the high surface albedo of the completely sea ice-covered oceans and the resulting very low temperatures. The main goal of this paper is to study the zonal and meridional momentum balances of the snowball Earth Hadley cell. This enables us to check the validity of prominent dry Hadley cell theories for virtually dry snowball Earth conditions, and by this to assess the assumptions that enter these theories. Note that in this study we restrict attention to the case of equinox insolation, but we intend to repeat this study for solstice and seasonally varying insolation as outlined in the discussion section.

In this paper, we focus on the two most prominent classes of dry Hadley cell theories. The first class comprises so-called axisymmetric, nearly inviscid theories that were pioneered by Schneider (1977) and Held and Hou (1980) and recently were extended by Caballero et al. (2008). Assuming that zonal-mean angular momentum in the upper Hadley cell branch is conserved, and combining this with thermal wind balance and the constraint of zero energy export from the Hadley cell region, these theories predict the Hadley cell strength and width in dependence of external parameters such as rotation rate and radiative equilibrium pole-toequator temperature contrast (e.g., Held and Hou 1980; Schneider 2006).

Dropping the assumption of purely axisymmetric flow and including eddies and their momentum and heat transports yields the second class of Hadley cell theories (Schneider 2006) on which we focus in this paper. Including eddies is important for a complete Hadley cell theory since they are found to be important in the upper Hadley cell branch of the modern Earth atmosphere (Walker and Schneider 2006). Theories of this class do not possess the neat feature of being analytically solvable since eddy fluxes are a priori unknown and depend on the zonal mean circulation. However, one can use idealized general circulation models to investigate dry Hadley cells (Becker et al. 1997; Walker and Schneider 2006) and to propose scaling laws for the dry Hadley cell

\footnotetext{
${ }^{1}$ Note that while Williams (1988a) described simulations of what he called "dry" atmospheres, these simulations did not model real dry atmospheres because they used climatological water vapor for radiation and "pseudo-dry" convective adjustment that relaxed to the moist instead of the dry adiabat.
}

strength and width (e.g., Walker and Schneider 2006; Korty and Schneider 2008).

The paper is organized as follows. Section 2 describes the AGCM ECHAM5 used in this study. This description focuses on the comprehensive suite of physical parameterizations of this AGCM that distinguishes our study from previous studies with idealized AGCMs. Section 2 also outlines the simulation setup. Section 3 summarizes the main features of the zonal-mean circulation of the snowball Earth atmosphere. Section 4 analyzes the zonal and meridional momentum balances. Section 5 investigates the effect of vertical diffusion of momentum on the strength of the snowball Earth Hadley cell. Section 6 discusses the implication of our results for dry Hadley cell theories. Section 7 follows with conclusions. In the appendix we show that the zonal and meridional momentum balances are not substantially altered when the diurnal insolation cycle is included and the model's parameterization of eddy viscosity and diffusivity for stable and neutral conditions is changed.

\section{Model and simulation setup}

We use the Max Planck Institute for Meteorology atmospheric general circulation model ECHAM5.4.01 (hereafter ECHAM5). ECHAM5 is a spectral hydrostatic model supplemented with comprehensive physics parameterizations solved in gridpoint space on a Gaussian grid. The model is comprehensively documented by Roeckner et al. (2003). We here focus on those physics parameterizations that distinguish ECHAM5 from previously used idealized AGCMs.

ECHAM5 comprises a full radiation scheme with 6 spectral bands in the shortwave and 16 bands in the longwave region. Longwave radiation is calculated by the Rapid Radiative Transfer Model (RRTM; Mlawer et al. 1997). ECHAM5 contains separate schemes for moist and dry convection. Moist convection follows Tiedtke (1989) and Nordeng (1994) but is of little importance for the cold and virtually dry snowball Earth conditions. Dry convection will turn out to be important to the snowball Earth Hadley cell, so we briefly summarize the dry convection (or turbulence) scheme here. Turbulent surface fluxes of momentum and heat are calculated from bulk aerodynamic formulas with transfer coefficients that are obtained from Monin-Obukhov similarity theory and that depend on stability through the "moist" bulk Richardson number (Brinkop and Roeckner 1995). Above the surface layer, the time tendencies due to the vertical turbulent fluxes of horizontal momentum $u$ and $v$ and dry static energy $s$ are modeled by vertical diffusion following the eddy diffusion method: 


$$
\begin{aligned}
& {\frac{\partial u}{\partial t}{ }_{\text {diff }}}^{=}=\rho g^{2} \frac{\partial}{\partial p} \rho^{2} K_{m} \frac{\partial u}{\partial p}, \\
& \frac{\partial v}{\partial t_{v \text { diff }}}=\rho g^{2} \frac{\partial}{\partial p} \rho^{2} K_{m} \frac{\partial v}{\partial p} \\
& {\frac{\partial s}{\partial t} v_{\text {diff }}}^{=}=\rho g^{2} \frac{\partial}{\partial p} \rho^{2} K_{s} \frac{\partial s}{\partial p}
\end{aligned}
$$

with density $\rho$, Earth's gravitational acceleration $g$, pressure $p$, and diffusion coefficients $K_{m}$ and $K_{s}$. Following standard terminology, we will refer to $K_{m}$ and $K_{s}$ as eddy viscosity and eddy diffusivity, respectively. By using the eddy diffusion method, the dry convection scheme assumes that unresolved eddies transport horizontal momentum and dry static energy in the direction of the vertical gradient of the resolved flow, which we will discuss in section 6. Using a 1.5-level closure scheme (Garratt 1992), eddy viscosity and diffusivity depend on the mixing length, the moist Richardson number, and turbulent kinetic energy (TKE). The dependence of eddy viscosity and diffusivity on the moist Richardson number is implemented by long-tail stability functions that are similar to Louis (1979). TKE is calculated by a simplified prognostic TKE equation that uses eddy viscosity for all turbulent fluxes (Roeckner et al. 2003; Brinkop and Roeckner 1995). The top of the boundary layer varies in time and space and is diagnosed as the maximum of two heights; the first height is based on the friction velocity and the second height is defined as the highest level with smaller dry static energy than the surface level. Note that the vertical transport of horizontal momentum and dry static energy by the dry convection scheme extends somewhat above the planetary boundary layer (PBL) owing to the model's parameterization of eddy viscosity and diffusivity.

We use ECHAM5 with idealized snowball Earth boundary conditions. These boundary conditions are not chosen to represent a potential Neoproterozoic snowball Earth (Kirschvink 1992; Hoffman et al. 1998; Hoffman and Schrag 2002), but to yield an idealized setup to study the snowball Earth Hadley cell. The surface is specified as sea ice everywhere with a surface heat capacity corresponding to 0.1-m-thick sea ice, implying small thermal inertia of the sea ice surface. The surface temperature is calculated from the balance of radiative (longwave and shortwave) and sensible and latent heat fluxes; conductive heat flux through sea ice is set to zero. Our setup therefore is equivalent to a thin "white" mixed-layer ocean with zero ocean heat transport. Surface roughness length is $10^{-3} \mathrm{~m}$ everywhere. Atmospheric $\mathrm{CO}_{2}$ content is specified to $300 \mathrm{ppmv} ; \mathrm{CH}_{4}, \mathrm{~N}_{2} \mathrm{O}$, chlorofluorocarbons (CFCs), ozone, and aerosols are all set to zero. A modern Earth total solar irradiance of $1365 \mathrm{~W} \mathrm{~m}^{-2}$ and a circular
TABLE 1. Summary of ECHAM5 simulations. Labels indicate whether vertical diffusion of zonal momentum (U), meridional momentum (V), and/or TKE (T) follows the standard setup of ECHAM5 $($ on $=1)$ or is suppressed above $870 \mathrm{hPa}($ off $=0)$. The Hadley cell strength is measured by the absolute maximum of the mass streamfunction $\left(10^{9} \mathrm{~kg} \mathrm{~s}^{-1}\right)$.

\begin{tabular}{lcccc}
\hline \hline Simulation & $\begin{array}{c}\text { Zonal } \\
\text { momentum }\end{array}$ & $\begin{array}{c}\text { Vertical diffusion } \\
\text { of meridional } \\
\text { momentum }\end{array}$ & TKE & $\begin{array}{c}\text { Hadley } \\
\text { cell } \\
\text { strength }\end{array}$ \\
\hline STD & On & On & On & 76 \\
U0V0T1 & Off & Off & On & 172 \\
U0V0T0 & Off & Off & Off & 121 \\
U0V1T1 & Off & On & On & 70 \\
U0V1T0 & Off & On & Off & 60 \\
U1V0T1 & On & Off & On & 214 \\
U1V0T0 & On & Off & Off & 164 \\
\hline
\end{tabular}

orbit with zero obliquity are applied. Motivated by the fact that Hadley cell theories do not incorporate the diurnal insolation cycle, daily-averaged insolation is used. This results in permanent equinox insolation conditions with maximum insolation of $434 \mathrm{~W} \mathrm{~m}^{-2}$ at the equator and zero insolation at the poles. Horizontal resolution is T63 $\left(1.75^{\circ}\right)$ with 31 unevenly spaced hybrid sigma levels in the vertical.

After initialization, the model is integrated for $20 \mathrm{yr}$. Statistics are taken from the last 5 yr. As the Northern and Southern Hemispheres are statistically identical in our simulations, results are averaged over both hemispheres. In all figures, if the sign of a plotted quantity depends on the hemisphere, the Northern Hemisphere sign of this quantity is chosen. Table 1 summarizes the simulations.

\section{Time-mean, zonal-mean potential temperature and circulation}

Running ECHAM5 in the idealized equinox snowball Earth conditions leads to a cold and virtually dry atmosphere owing to the high surface albedo (simulation STD). The global-mean surface temperature is $202 \mathrm{~K}$ with chilly equatorial surface temperatures of $220 \mathrm{~K}$. The steep meridional insolation gradient produces an equatorto-pole surface temperature contrast of $90 \mathrm{~K}$ (Fig. 1). This is about twice as large as for modern Earth (Peixoto and Oort 1992). The global-mean water vapor content of $7 \times 10^{-3} \mathrm{~kg} \mathrm{~m}^{-2}$ is about three orders of magnitudes smaller than in modern Earth's atmosphere.

Equatorward of $15^{\circ}$, potential temperature decreases with height in the immediate vicinity of the surface, a manifestation of persistent static instability in the surface layer, and is otherwise uniform below $600 \mathrm{hPa}$. Strong vertical gradients above $550 \mathrm{hPa}$ illustrate stable stratification in the upper equatorial atmosphere. Meridional potential temperature gradients in the lower atmosphere increase rapidly toward the poles and are accompanied by 

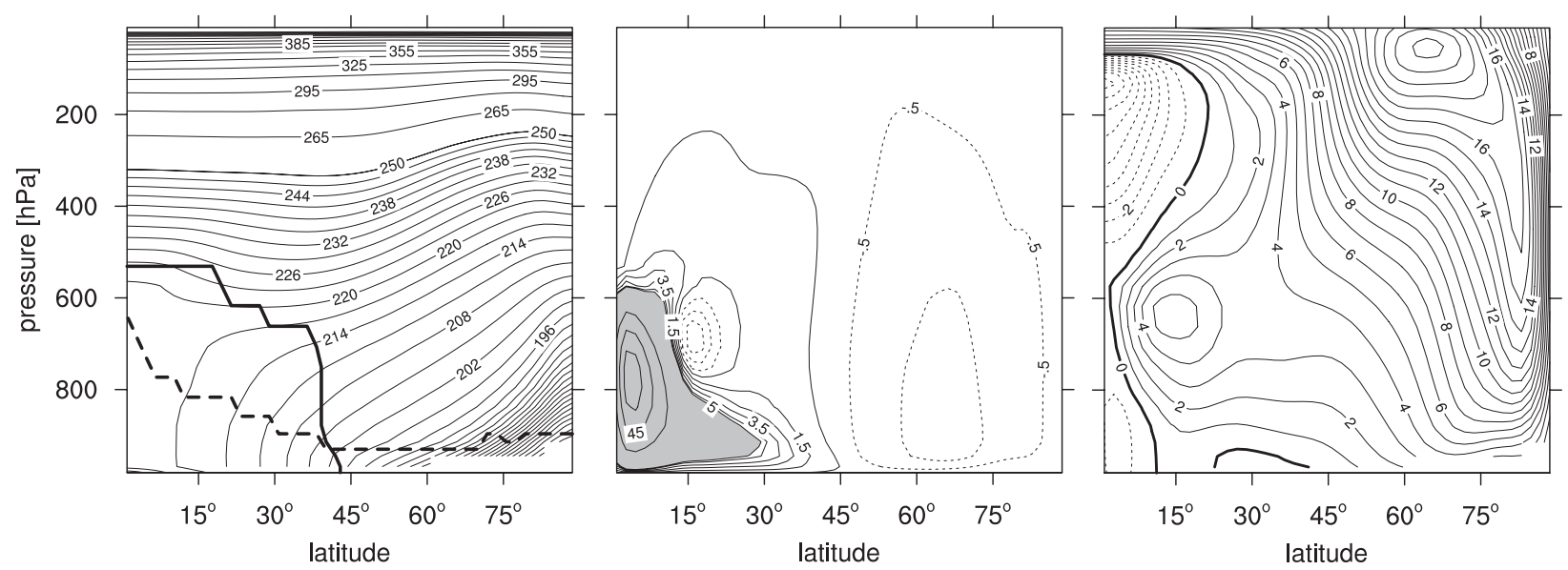

FIG. 1. Time-mean, zonal-mean structure of the idealized equinox snowball Earth atmosphere (simulation STD). (left) Potential temperature $(\mathrm{K})$. Contour interval $(\mathrm{CI})$ is $3 \mathrm{~K}$ below $250 \mathrm{~K}$ and $15 \mathrm{~K}$ above; the black solid line depicts the tropopause (see text) and the black dashed line shows the top of the PBL. (middle) Mass streamfunction $\left(10^{9} \mathrm{~kg} \mathrm{~s}^{-1}\right)$. Solid (dashed) contour lines represent clockwise (counterclockwise) circulation. CI is $20 \times 10^{9} \mathrm{~kg} \mathrm{~s}^{-1}$ above $5 \times 10^{9} \mathrm{~kg} \mathrm{~s}^{-1}$ (gray shading) and $1 \times 10^{9} \mathrm{~kg} \mathrm{~s}^{-1}{\mathrm{below} 5 \times 10^{9} \mathrm{~kg} \mathrm{~s}}^{-1}$. (right) Zonal wind $\left(\mathrm{m} \mathrm{s}^{-1}\right)$. CI is $1 \mathrm{~m} \mathrm{~s}^{-1}$, with a thick zero contour line.

steep vertical gradients poleward of $45^{\circ}$. The latter reflect a lower-atmosphere temperature inversion poleward of $45^{\circ}$.

A lapse-rate criterion of $4 \mathrm{~K} \mathrm{~km}^{-1}$ puts the tropical tropopause at $550 \mathrm{hPa}$ (Fig. 1). This is in excellent agreement with the estimate of the tropopause in optically thin atmospheres given in Pierrehumbert (2010). After a slow decrease in height between $20^{\circ}$ and $40^{\circ}$ latitude, the tropopause drops to the surface poleward of $45^{\circ}$ because of the lower-atmosphere temperature inversion there. The planetary boundary layer extends to $650 \mathrm{hPa}(\sim 2700 \mathrm{~m})$ at the equator and, in contrast to modern Earth, almost coincides with the equatorial tropopause. This is a consequence of the lack of moist convection, which on modern Earth permits the separation of the tropical tropopause and the PBL. Between $5^{\circ}$ and $40^{\circ}$ latitude, the planetary boundary layer ends about $200 \mathrm{hPa}$ below the tropopause, again much less than on modern Earth. Poleward of $45^{\circ}$ the boundary layer extends beyond the tropopause. There, the top of the planetary boundary layer is decoupled from the vertical profile of dry static energy and is set by the friction velocity.

The meridional circulation consists of a direct clockwise cell (seen from the east for the Northern Hemisphere) from the equator to $45^{\circ}$, and an indirect counterclockwise cell poleward thereof (Fig. 1). The direct cell is subdivided into a weak deep stratospheric cell and a much stronger tropospheric cell. In the remainder of this paper, we will refer to this tropospheric cell as the snowball Earth Hadley cell, or simply as the Hadley cell. The snowball Earth Hadley cell attains its maximum inside the planetary boundary layer and is about equal in strength to the annual-mean Hadley cell of today's Earth. While the bulk of the transport is realized equatorward of $15^{\circ}$ latitude, the snowball Earth Hadley cell exhibits a weak low-level extension to $45^{\circ}$ latitude. These features make the snowball Earth Hadley cell similar to the dry Hadley cell in the simulations of Frierson et al. (2006). Moreover, note that a deep stratospheric cell was also seen in the axisymmetric model of Caballero et al. (2008). However, in contrast to Caballero et al. (2008), our deep cell does not conserve zonal-mean angular momentum (see section 4). Below the tropopause at the upper poleward edge of the snowball Earth Hadley cell, a weak counterclockwise cell appears in the upper troposphere at around $15^{\circ}$ latitude.

The surface zonal wind has, going from the equator to the pole, an easterly-westerly-easterly-westerly pattern (Fig. 1). Poleward of $45^{\circ}$, strong vertical shear of zonal wind and meridional potential temperature gradients indicate high eddy activity that results in a jet at $65^{\circ}$ latitude and $50 \mathrm{hPa}$. In the upper Hadley cell branch, the zonal-mean zonal wind is much too weak to conserve zonal-mean angular momentum (Schneider 2006). This is due to zonal-mean angular momentum sinks by eddies as well as vertical diffusion, as analyzed in the next section.

\section{Hadley cell dynamics}

To investigate which processes are important for the idealized equinox snowball Earth Hadley cell, we analyze the time-mean, zonal-mean zonal and meridional momentum balances.

\section{a. Zonal momentum balance}

In spherical pressure coordinates and after taking the time and zonal mean, the steady-state primitive zonal momentum balance reads 

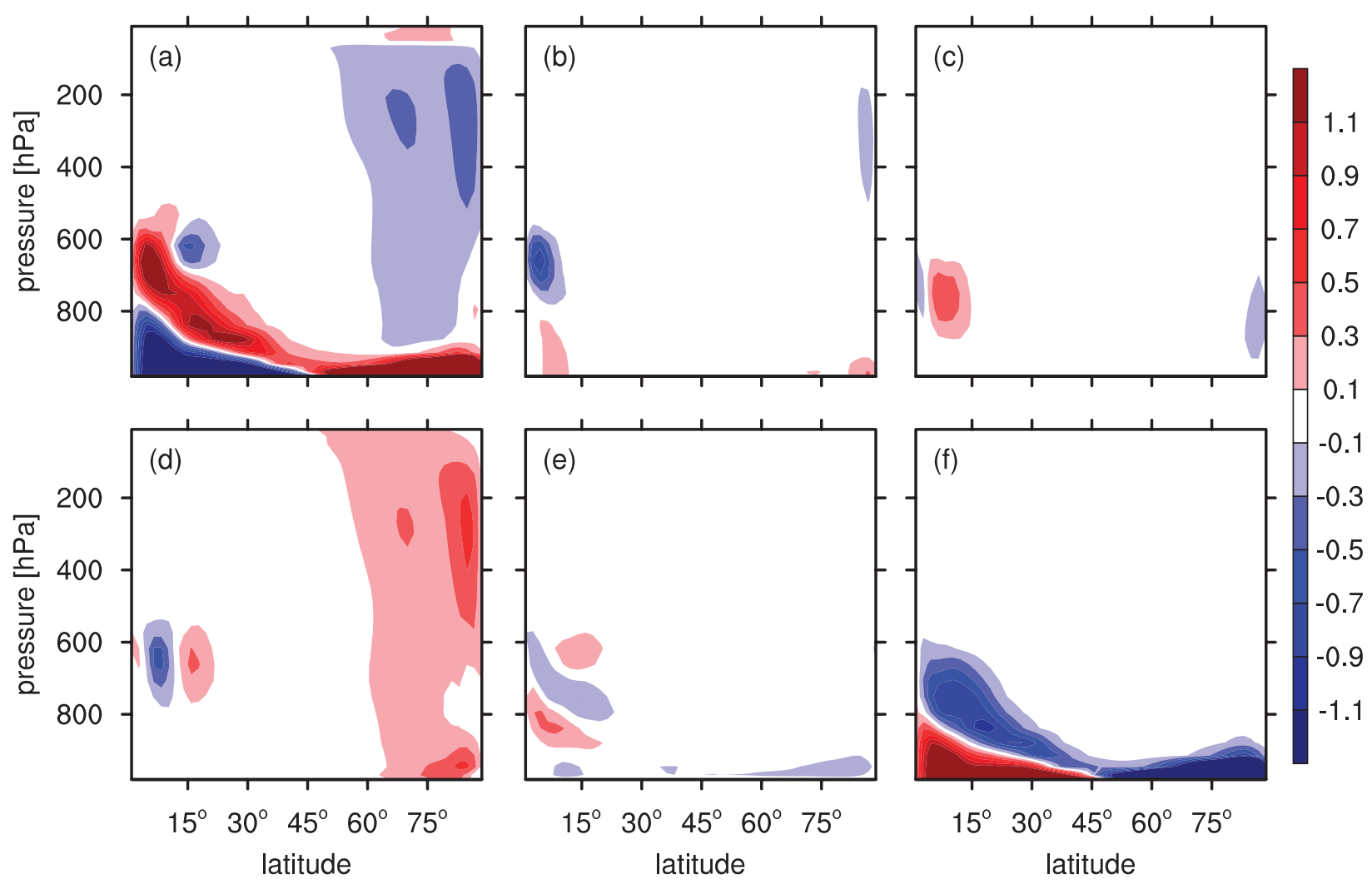

FIG. 2. Time-mean, zonal-mean zonal momentum balance of the idealized equinox snowball Earth atmosphere $\left(10^{-5} \mathrm{~m} \mathrm{~s}^{-2} ;\right.$ simulation STD): (a) Coriolis force, (b) meridional advection of mean relative vorticity, (c) negative of vertical advection of mean zonal momentum, (d) horizontal contribution to eddy momentum flux convergence, (e) vertical contribution to eddy momentum flux convergence, and (f) vertical diffusion of zonal momentum. This order corresponds to the order of the terms of the rhs of Eq. (1). The contributions of cumulus friction and horizontal diffusion are negligible.

$$
\begin{aligned}
\frac{\partial \bar{u}}{\partial t}= & 0=f \bar{v}+\bar{\zeta} \bar{v}-\bar{\omega} \frac{\partial \bar{u}}{\partial p}-\frac{1}{a \cos ^{2} \varphi} \frac{\partial\left(\overline{u^{\prime} v^{\prime}} \cos ^{2} \varphi\right)}{\partial \varphi} \\
& -\frac{\partial \overline{u^{\prime} w^{\prime}}}{\partial p}+\bar{F}^{u} .
\end{aligned}
$$

Here, $f$ is the Coriolis parameter; $u, v$, and $\omega$ respectively label the zonal, meridional, and pressure velocities; $\zeta$ is the relative vorticity; $a$ is Earth's radius; $\varphi$ is latitude; and $p$ is pressure. Overbars denote time-mean, zonal-mean quantities, and primes deviations thereof. Also, $\bar{F}^{u}=\bar{F}_{v \text { diff }}^{u}+\bar{F}_{\text {cfr }}^{u}+\bar{F}_{h \text { diff }}^{u}$ summarizes zonal momentum tendencies due to parameterized processes. This comprises, in the following order, vertical diffusion of zonal momentum (including surface drag) as part of the dry convection scheme, cumulus friction, and horizontal diffusion. For our analysis, we calculate mean and eddy terms based on $(u, v, \omega, \zeta)$ interpolated to 31 pressure levels that are close to the model's 31 hybrid sigma levels. Tendencies due to the parameterized processes are diagnosed during the model run for each grid point, hybrid sigma level, and time step, interpolated to pressure levels

using the same interpolation algorithm as for $(u, v, \omega, \zeta)$, and finally averaged over time and longitude.

In the lowest part of the atmosphere, the Coriolis force is primarily balanced by vertical diffusion of zonal momentum (Fig. 2). Away from the surface, the zonal momentum balance in the counterclockwise cell poleward of $45^{\circ}$ is between the Coriolis force and the meridional eddy momentum flux. In the tropics, the zonal momentum balance of the snowball Earth atmosphere differs substantially from that of modern Earth. For modern Earth, the upper Hadley cell branch shows a balance between mean meridional advection of mean absolute vorticity and eddy momentum flux divergence $S$ (Walker and Schneider 2006):

$$
(f+\bar{\zeta}) \bar{v} \approx S .
$$

In the snowball Earth atmosphere this balance carries over for the deep weak cell above $550 \mathrm{hPa}$ (not visible in Fig. 2 because of the chosen contour spacing), but does not hold in the upper branch of the Hadley cell. There, vertical diffusion of zonal momentum is essential to the 
zonal momentum balance, which can be approximated by

$$
(f+\bar{\zeta}) \bar{v} \approx S-\bar{F}_{v \text { diff }}^{u}
$$

This balance has two implications. First, zonal-mean angular momentum is not conserved in the upper branch of the snowball Earth Hadley cell. Second, the upper branch of the snowball Earth Hadley cell is not exclusively controlled by large-scale dynamics but is also affected by the parameterized process of dry convection, showing up as vertical diffusion of zonal momentum.

\section{b. Meridional momentum balance}

In contrast to the zonal momentum balance, the steady-state primitive meridional momentum equation in spherical pressure coordinates and averaged by time and longitude contains the geopotential gradient. As a consequence and similar to modern Earth, the Coriolis force and the geopotential gradient dominate the meridional momentum balance in most regions of the snowball Earth atmosphere, implying that zonal winds are mostly in thermal wind balance. In the Hadley cell region, however, vertical diffusion of meridional momentum is important. Vertical diffusion decelerates the meridional momentum in the upper branch of the Hadley cell and accelerates the meridional momentum in the lower branch (Fig. 3). This reduces the vertical shear of meridional momentum by downgradient momentum mixing, and the zonal wind deviates from thermal wind balance in the region of the snowball Earth Hadley cell. The meridional momentum budget there reads

$$
f \bar{u} \approx-\frac{1}{a} \frac{\partial \bar{\Phi}}{\partial \varphi}+\bar{F}_{v \mathrm{diff}}^{v}(p, \varphi) .
$$

Here, $\Phi$ denotes the geopotential and $F_{v \text { diff }}^{v}$ labels vertical diffusion of meridional momentum. Test runs have shown that the influence of vertical diffusion in the zonal as well as the meridional momentum balance does not change when the number of vertical levels is doubled.

\section{Influence of vertical diffusion of momentum on Hadley cell strength}

Motivated by the fact that the two classes of dry Hadley cell theories described in the introduction assume zero vertical diffusion of momentum in the Hadley cell's upper branch, we investigate how the strength of the snowball Earth Hadley cell changes when vertical diffusion of momentum is suppressed in its upper branch. The momentum balances propose two competing effects of

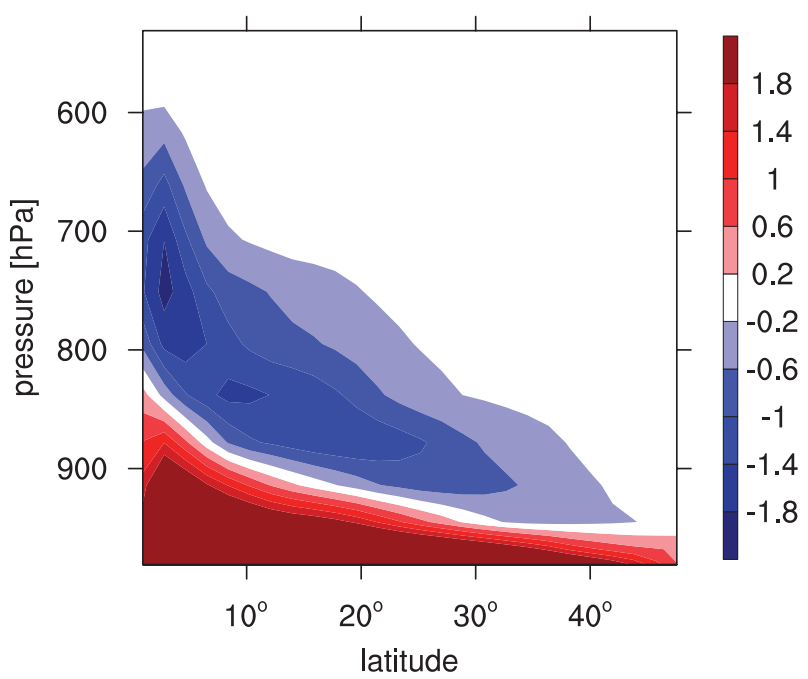

FIG. 3. Meridional momentum tendency due to vertical diffusion of meridional momentum of the idealized equinox snowball Earth atmosphere $\left(10^{-5} \mathrm{~m} \mathrm{~s}^{-2}\right.$; simulation STD).

vertical diffusion of momentum on the Hadley cell strength. On the one hand, the zonal momentum balance suggests that suppressing vertical diffusion of zonal momentum in the upper branch should result in a weaker Hadley cell. Because $\bar{F}_{v \text { diff }}^{u}$ is negative in the upper branch, setting $\bar{F}_{v \text { diff }}^{u}$ to zero must lead to a decrease of the zonalmean meridional velocity in the upper branch and thereby a weaker Hadley cell if we assume that all other terms remain the same [cf. Eq. (3)]. On the other hand, the meridional momentum balance suggests that suppressing vertical diffusion of meridional momentum should result in a stronger Hadley cell since this eliminates the decelerating meridional momentum mixing between its upper and lower branches. These considerations neglect that when vertical diffusion of momentum is suppressed, other terms of the momentum balance will adjust as well. Therefore, there is no guarantee that the response of the Hadley cell to suppressed vertical diffusion of momentum follows these considerations, and an analysis using a diagnostic equation for the mean meridional circulation (Kim and Lee 2001a,b) would certainly be helpful. However, as we show below, the response of the Hadley cell is indeed in line with these two considerations.

To suppress vertical diffusion of zonal and meridional momentum in the upper branch of the snowball Earth Hadley cell, we set eddy viscosity to zero above $870 \mathrm{hPa}$. This is readily implemented but introduces an ambiguity in the vertical diffusion of TKE, which uses eddy viscosity as diffusion coefficient (see section 2) and affects the vertical diffusion of dry static energy through the parameterization of eddy diffusivity. Since it is unclear to us whether vertical diffusion of TKE should be done 

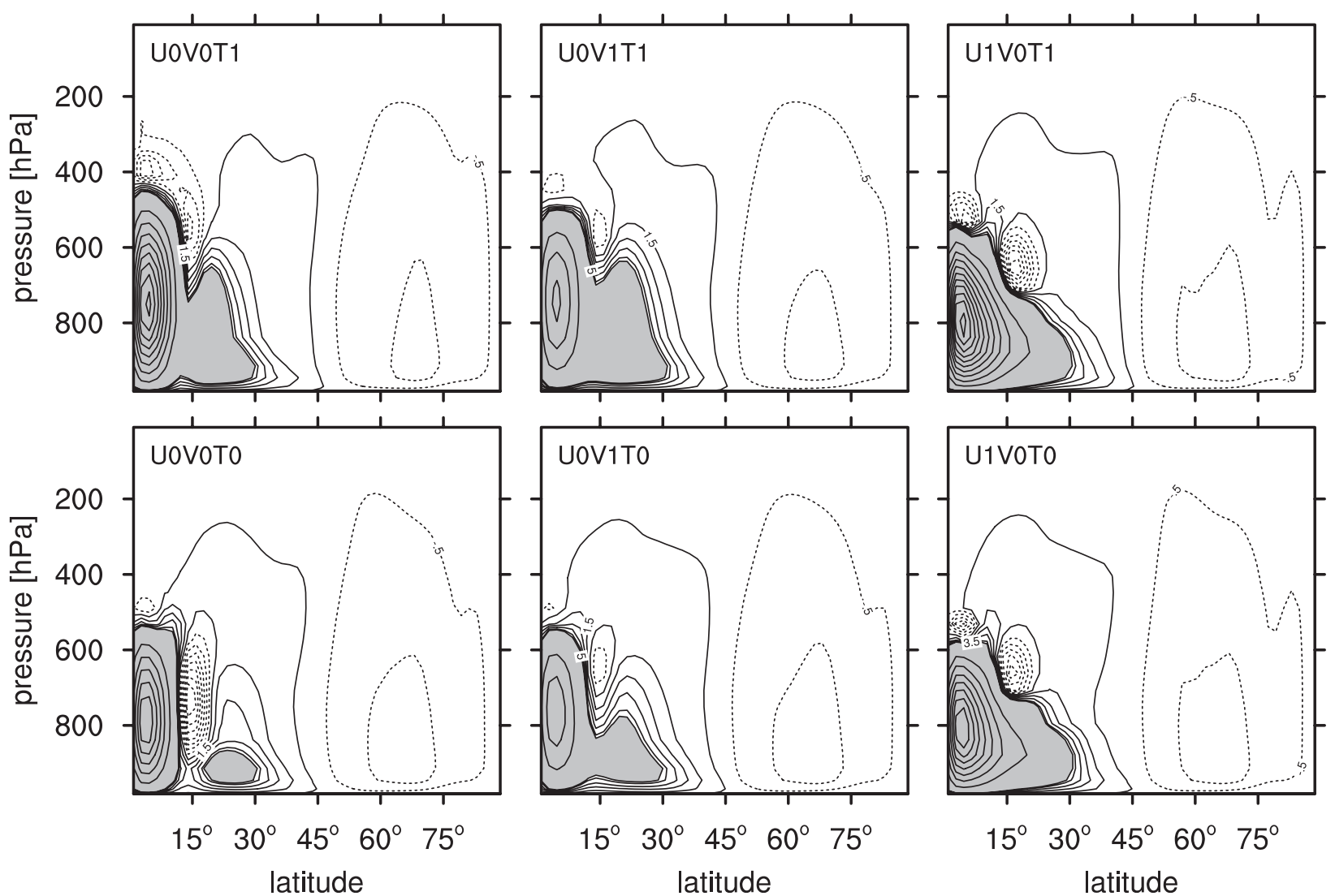

FIG. 4. Mass streamfunction $\left(10^{9} \mathrm{~kg} \mathrm{~s}^{-1}\right)$ when vertical diffusion is suppressed above $870 \mathrm{hPa}$ for (left) both zonal and meridional momentum, (middle) only zonal momentum, and (right) only meridional momentum, for simulations with (top) full and (bottom) suppressed vertical diffusion of TKE. Solid (dashed) contour lines represent clockwise (counterclockwise) circulation. Contour interval is $20 \times 10^{9} \mathrm{~kg} \mathrm{~s}^{-1}$ above $5 \times 10^{9} \mathrm{~kg} \mathrm{~s}^{-1}$ (gray filling), and $1 \times 10^{9} \mathrm{~kg} \mathrm{~s}^{-1}$ below $5 \times 10^{9} \mathrm{~kg} \mathrm{~s}^{-1}$.

with the originally computed full eddy viscosity or the restricted eddy viscosity enforced to zero above $870 \mathrm{hPa}$, and to make sure that the response of the Hadley cell does not crucially depend on this choice, we perform simulations with one or the other choice. To check if the response of the Hadley cell follows the two considerations described above, we also perform simulations in which we only suppress vertical diffusion of either zonal or meridional momentum. We do this by using the restricted eddy viscosity for the vertical diffusion of one of the horizontal momentum components while using the full eddy viscosity for the vertical diffusion of the other component. We do not explicitly change the eddy diffusivity used for the vertical diffusion of dry static energy, but changes in the wind and temperature field as well as TKE that result from the change in eddy viscosity may lead to changes in eddy diffusivity and vertical diffusion of dry static energy compared to the control case STD.

Suppressing vertical diffusion of zonal and meridional momentum above $870 \mathrm{hPa}$ roughly doubles the Hadley cell strength, depending somewhat on the choice of eddy viscosity for vertical diffusion of TKE. As anticipated from the momentum balances, suppressing vertical diffusion of zonal momentum weakens the Hadley cell whereas suppressing vertical diffusion of meridional momentum strengthens the Hadley cell (Fig. 4; Table 1). However, the weakening for suppressed vertical diffusion of zonal momentum is small compared to the strengthening for suppressed vertical diffusion of meridional momentum. When vertical diffusion of zonal momentum is suppressed, the divergence of the vertical eddy momentum flux becomes much stronger and changes such as to compensate for the loss of vertical diffusion of zonal momentum in the zonal momentum balance. As an example, this is shown in Fig. 5 for simulation U0V1T1. The vertical eddy momentum flux appears to attenuate the weakening of the Hadley cell when vertical diffusion of zonal momentum is suppressed. As a result, vertical diffusion of meridional momentum exhibits a stronger control on the Hadley cell strength than does vertical diffusion of zonal momentum. 

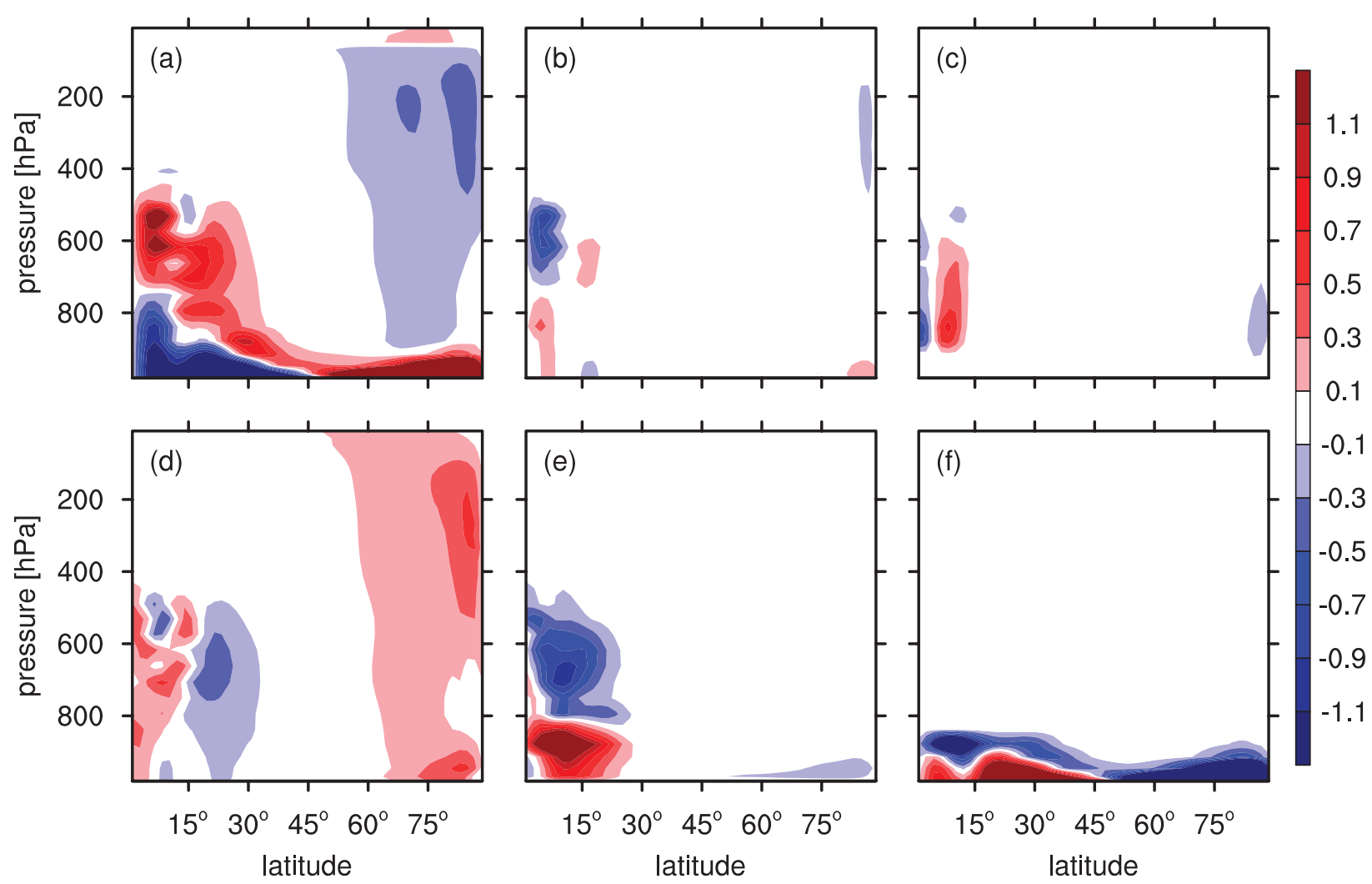

FIG. 5. As in Fig. 2, but when vertical diffusion of zonal momentum is suppressed above $870 \mathrm{hPa}$ (simulation U0V1T1).

\section{Discussion}

Our simulations show that vertical diffusion of momentum plays a major role for the dynamics of the idealized equinox snowball Earth Hadley cell. Since this Hadley cell is an example of a (virtually) dry atmosphere, this has important implications for dry Hadley cells in general as discussed in the following.

Zonal-mean angular momentum is not conserved in the upper branch of the snowball Earth Hadley cell due to the influence of eddies as well as vertical diffusion. This implies that axisymmetric Hadley cell theories based on conservation of zonal-mean angular momentum (e.g., Held and Hou 1980; Caballero et al. 2008) are not applicable to this atmosphere. Interestingly, Caballero et al. (2008) already speculated that vertical diffusion of momentum, or vertical transport of momentum by dry convection, might be important for dry Hadley cells. Moreover, in contrast to what is sometimes assumed in axisymmetric Hadley cell theories (Held and Hoskins 1985), the meridional mass transport of the snowball Earth Hadley cell is not confined to well-separated upper and lower branches. Instead, there is no significant region of nearly vanishing meridional flow in the center of the snowball Earth Hadley cell. This leads to strong meridional wind shear and makes diffusive transfer of meridional momentum between upper and lower branches possible if not inevitable.

Our simulations also differ from axisymmetric simulations with respect to the influence of vertical diffusion of momentum on the Hadley cell strength. In axisymmetric simulations, which typically use a globally uniform viscosity, decreasing viscosity weakens the Hadley cell (e.g., Held and Hou 1980; Hou 1984; Becker et al. 1997; Kim and Lee 2001b), in line with what one expects from the zonal momentum balance. In contrast, our comprehensive AGCM exhibits a doubling of the Hadley cell strength when vertical diffusion of momentum is suppressed in the upper branch of the cell. While caution is mandatory when comparing these axisymmetric simulations to our results because our simulations do not apply globally uniform eddy viscosity and because we set eddy viscosity to zero above $870 \mathrm{hPa}$ instead of decreasing it globally, we speculate that one reason for this difference is the presence of vertical eddy momentum transport in our comprehensive AGCM. By compensating, at least partly, for the loss of vertical diffusion of zonal momentum, the vertical eddy momentum transport weakens the influence of vertical diffusion of zonal momentum on the Hadley cell strength in our simulations. 
In contrast to the atmosphere of modern Earth, the zonal momentum balance in the upper branch of the snowball Earth Hadley cell is not dominated by largescale dynamics through mean flow advection and eddy momentum fluxes, but involves vertical diffusion of zonal momentum. This calls for reconsideration of the dry Hadley cell results of Walker and Schneider (2006) and Korty and Schneider (2008) because their idealized AGCM (see Schneider and Walker 2006) fixed the planetary boundary layer at $2.5 \mathrm{~km}(\simeq 850 \mathrm{hPa})$ and neglected vertical transport by dry convection above $2.5 \mathrm{~km}$, despite unstable background stratification and active dry convection up to $400 \mathrm{hPa}$. By design, this idealized AGCM hence assumes that the upper Hadley cell branch is in the free troposphere, but the exclusion of momentum mixing by dry convection in this idealized model appears too strong a simplification.

We find that in the snowball Earth atmosphere, the tropopause and the planetary boundary layer are separated by at maximum $250 \mathrm{hPa}$, leaving only little space for a free troposphere. This renders the snowball Earth atmosphere very different from modern Earth and points at a fundamental question for dry atmospheres: do dry atmospheres manage to separate the tropical tropopause from the top of the PBL, and if so, how and to what extent? Since longwave radiative cooling destabilizes the troposphere (Pierrehumbert 2010), other processes need to provide the necessary stabilizing heating. In moist atmospheres, this task is accomplished by moist convection through latent heat release, but this mechanism is not available in dry atmospheres. If we further restrict attention to dry atmospheres that are transparent to solar radiation, which is a good approximation to the snowball Earth atmosphere (as well as the Martian atmosphere) if no atmospheric dust is present, the heating can only be achieved by some combination of dry convection and large-scale temperature advection. While the intensity of dry convection should be inversely related to the ability of the large-scale circulation to provide the heating, the exact partitioning of the heating between the two is not obvious. Interestingly, in the dry limit of the idealized AGCM of Frierson et al. (2006), which includes a dynamic planetary boundary layer and momentum mixing by dry convection, the tropical tropopause and PBL top nearly coincide. However, as pointed out by Caballero et al. (2008), if the Hadley cell is to transport energy meridionally, potential temperatures in the Hadley cell cannot be perfectly uniform in the vertical. In Caballero et al. (2008), potential temperatures in the upper part of the subsiding region of the Hadley cell are warmer than they would be on a dry adiabat; this showed up as a poleward tilt of the isentropes there. We see a similar poleward tilt in our simulations, and, as in

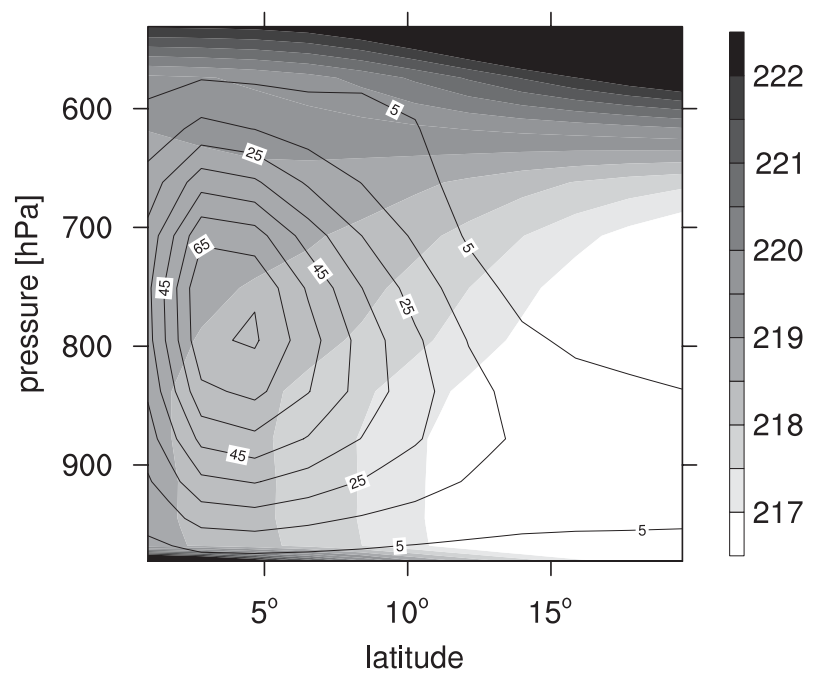

FIG. 6. Close-up of the time-mean, zonal-mean potential temperature (shading, K) and mass streamfunction (contour lines, $10^{9} \mathrm{~kg} \mathrm{~s}^{-1}$ ) of the idealized equinox snowball Earth atmosphere (simulation STD). Contour interval for potential temperature is $0.5 \mathrm{~K}$. Contour interval for the mass streamfunction is $10 \times 10^{9}$ $\mathrm{kg} \mathrm{s}^{-1}$; the solid contour lines indicate clockwise circulation.

Caballero et al. (2008), this tilt is created by the Hadley cell itself through horizontal and vertical potential temperature advection (Fig. 6). The Hadley cell therefore generates some static stability in the subsiding branch. While in our simulations, this is not sufficient to eliminate dry convection in the subsiding branch, it might be an important mechanism in limiting dry convection for stronger dry Hadley cells.

Our results show that studying dry Hadley cell dynamics requires a model that resolves not only the largescale circulation but also vertical momentum transport by dry convection (as done by vertical diffusion in our model) and that includes planetary boundary layer dynamics. Idealized AGCMs and dry Hadley cell theories that neglect vertical transport of momentum by dry convection should not be considered as models or theories for real dry atmospheres but should be viewed as mechanistic tools to study the role of mean flow-eddy interactions for Hadley cells. A similar point of consideration applies to the representation of radiation. Since the strength of dry convection is linked to the rate of radiative destabilization by tropospheric longwave cooling, the radiation scheme is important for the results as well. The latter suggests that when radiation is modeled by Newtonian relaxation, the choice of the background temperature strongly impacts the Hadley cell dynamics. Our results and the results of Haqq-Misra et al. (2011) show that the particular choice of parameterizing unresolved processes such as dry convection or radiation can have significant impact on the circulation. 
The absence of water from dry atmospheres eliminates important challenges for moist Hadley cell theories, namely moist convection and its associated vertical transport of momentum, latent heat release, and cloud formation. Nevertheless, developing a Hadley cell theory for dry atmospheres comes with its own difficulties due to the need to incorporate dry convection and the associated vertical transport of momentum. The AGCM used in our study models this transport by vertical diffusion assuming a particular parameterization for eddy viscosity and diffusivity, while there are various other parameterizations for eddy viscosity and diffusivity (see, e.g., Stull 1988). In the appendix, we show that vertical transport of momentum by dry convection stays important when we alter the models parameterization of eddy viscosity and diffusivity for stable and neutral conditions. However, by modeling dry convection as vertical diffusion, our AGCM assumes that dry convection transports momentum and dry static energy down the local vertical gradient of the resolved flow (see section 2), while the transport might actually be upgradient, especially under convective conditions (Stull 1988). The latter has led to the development of nonlocal schemes that include countergradient transport terms (e.g., Holtslag and Boville 1993) or use mass-flux models to represent the transport by thermals (Hourdin et al. 2002; Soares et al. 2004). This variety illustrates that modeling dry convection and its associated transport is an extraordinarily difficult problem, and that large-eddy simulations that explicitly resolve at least part of the momentum transport by dry convection would be a helpful tool to quantify the momentum drag due to dry convection. In any case, our results show that the vertical transport of momentum due to dry convection needs to be taken seriously when one studies dry Hadley cell dynamics.

In this light, building a Hadley cell theory for realistic dry atmospheres might not be easier than for moist atmospheres. Moreover, because dry and moist convection potentially differ in how and at which magnitude they transport momentum vertically, obtaining a dry Hadley cell theory may only be of limited use as a stepping stone toward a moist Hadley cell theory. This is not to say that we should not attempt to develop a dry theory, since this dry theory would be of great help when studying the climate dynamics of dry planetary atmospheres. Also, the quest of framing a theory that reliably describes the Hadley cell over the wide range of past and possible future climates of Earth, which differ significantly in their atmospheric moisture content, will benefit from knowing the dry limit of this theory.

We close our discussion by mentioning three directions of future investigations. First, and most importantly, repeating our study for solstice insolation would clarify if dry convection and vertical diffusion of momentum are also important for dry Hadley cell dynamics when the heating is displaced away from the equator. This is motivated (a) by Walker and Schneider (2005), who suggest that the effect of eddies on the Hadley cell strength depends on the latitudinal insolation profile, (b) by the presence of a near-surface temperature inversion in the winter subtropics (Pierrehumbert et al. 2011) that might suppress dry convection in the subsiding branch of the winter Hadley cell, and (c) by indications that the Martian Hadley cell is close to the conservation of zonalmean angular momentum for solstice insolation (Haberle et al. 1993; Wilson 1997). Second, including seasonally varying insolation would allow us to reveal if dry Hadley cells undergo monsoonlike transitions also in a comprehensive AGCM, similar to what was found by Schneider and Bordoni (2008) in an idealized dry AGCM. Third, recent observations and reanalysis datasets of Earth's atmosphere showed the existence of a low-level, low-latitude circulation that is embedded into the conventional Hadley cell of Earth (Zhang et al. 2004, 2008). Idealized simulations reproduced these shallow circulations (Nolan et al. 2007). Since the upper branch of these shallow circulations is close to the planetary boundary layer, they seem similar to the snowball Earth Hadley cell.

\section{Conclusions}

Using the comprehensive atmospheric general circulation model ECHAM5 to study the Hadley circulation of an idealized snowball Earth atmosphere under equinox insolation, we draw the following conclusions:

- The upper branch of the snowball Earth Hadley cell is strongly influenced by vertical transport of momentum due to dry convection. In our model, this shows up as vertical diffusion of momentum in the zonal and meridional momentum balances. The dynamics of the snowball Earth Hadley cell are hence very different to the dynamics of the modern Earth Hadley cell. Neither axisymmetric Hadley cell models based on angular momentum conservation nor eddy-permitting Hadley cell models that neglect vertical transport of momentum by dry convection are applicable to the snowball Earth atmosphere.

- In our model, suppressing vertical momentum diffusion in the upper branch of the snowball Earth Hadley cell results in a doubling of the Hadley cell strength. This is consistent with the decelerating effect of vertical diffusion in the meridional momentum balance and shows that vertical diffusion of meridional momentum exhibits a stronger control on the snowball Earth Hadley cell than vertical diffusion of zonal momentum. 
TABLE A1. Summary of ECHAM5 simulations with diurnal insolation cycle (DIUR) and/or short-tail stability function for stable and neutral conditions (SHORT). The Hadley cell strength is measured by the absolute maximum of the mass streamfunction $\left(10^{9} \mathrm{~kg} \mathrm{~s}^{-1}\right)$.

\begin{tabular}{lccc}
\hline \hline \multicolumn{1}{c}{ Simulation } & $\begin{array}{c}\text { Diurnal } \\
\text { cycle }\end{array}$ & $\begin{array}{c}\text { Stability } \\
\text { functions }\end{array}$ & $\begin{array}{c}\text { Hadley cell } \\
\text { strength }\end{array}$ \\
\hline STD-DIUR & On & ECHAM5 & 104 \\
SHORT & Off & ZPK02 & 66 \\
SHORT-DIUR & On & ZPK02 & 103 \\
\hline
\end{tabular}

- Studying dry Hadley cells requires not only resolving the large-scale circulation but also incorporating vertical momentum transport by dry convection. This represents a significant and hitherto ignored complication to the development of dry Hadley cell theories.

Acknowledgments. We are grateful to the reviews by Raymond T. Pierrehumbert, Dorian S. Abbot, and one anonymous reviewer. We thank Bjorn Stevens for help with setting up the 62-level version of ECHAM5, Thorsten Mauritsen for help with implementing the shorttail stability function, and Jürgen Bader for comments and the internal review at MPI-M. This work was supported by the Max Planck Society and the International Max Planck Research School on Earth System Modelling. A.V. thanks the IMPRS-ESM Guest and Exchange Program for financial support for a visit to the Geophysical Fluid Dynamics Laboratory (GFDL) in Princeton, New Jersey, USA, and GFDL for their hospitality. All simulations were performed at the German Climate Computing Center (DKRZ) in Hamburg, Germany.

\section{APPENDIX}

\section{Additional Simulations}

This appendix presents additional simulations to verify that the importance of vertical diffusion of momentum on the idealized equinox snowball Earth Hadley cell is neither a consequence of using daily-averaged insolation nor a result of using a long-tail stability function in the parameterization of eddy viscosity. These simulations are motivated by two issues.

First, climate model studies showed that the planetary boundary layer over deserts exhibits a strong diurnal cycle (Medeiros et al. 2005; Randall et al. 1985). Despite a deep PBL during the day with maximum depth near sunset, the nocturnal PBL shrinking causes the time-mean PBL in simulations with a diurnal cycle of insolation to be shallower than in simulations that use daily-averaged insolation. Since the snowball Earth, like desert regions,

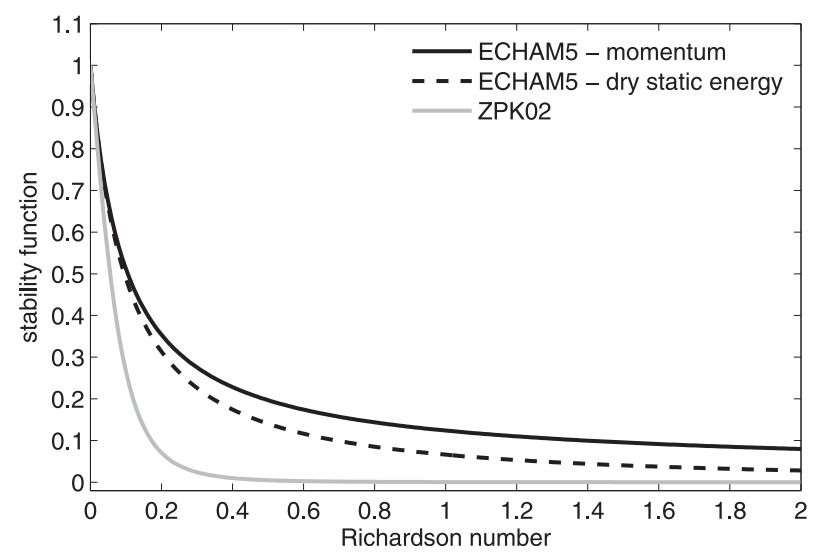

FIG. A1. The Louis-type long-tail stability functions used in ECHAM5 (black solid for momentum, black dashed for dry static energy) and the newly implemented short-tail stability function that falls rapidly to zero above $\mathrm{Ri}_{c}=0.2$ (ZPK02).

has a surface with low thermal inertia and dry air above, including the diurnal cycle might contract the time-mean PBL and therefore might decrease vertical diffusion of momentum in the upper branch of the snowball Earth Hadley cell.

Second, AGCMs used for numerical weather prediction and climate applications tend to generate too strong vertical diffusion in stable boundary layers compared to turbulence theory and observations. The strong diffusion is related to the choice of long-tail stability functions that do not drop to zero above the critical Richardson number $\mathrm{Ri}_{c}=0.2$, as suggested by Monin-Obukhov theory, but fall to zero slowly. In AGCMs, long-tail stability functions are used because they improve the simulations of the present-day climate (see Savijarvi 2009, and references therein). The stability function applied in ECHAM5 is of this long-tail type (see section 2).

We perform simulations with enabled diurnal cycle and/or a newly implemented short-tail stability function (Table A1). This short-tail stability function equals the standard stability function of ECHAM5 for unstable conditions but is much smaller for neutral and stable conditions. It follows the slight modification of Zilitinkevich et al. (2002) suggested by Savijarvi (2009):

$$
f_{\text {SHORT }}(\mathrm{Ri})=\left(1+5 \mathrm{Ri}+44 \mathrm{Ri}^{2}\right)^{-2},
$$

with Ri denoting the "moist" Richardson number. Here, $f_{\text {SHORT }}$ is the same for momentum and dry static energy (Fig. A1). In all other aspects, the simulations of this appendix are identical to simulation STD.

None of these changes causes a fundamentally different Hadley cell shape. As in STD, the bulk of mass streamfunction lies within $15^{\circ}$, with low-level extension of about $5 \times 10^{9} \mathrm{~kg} \mathrm{~s}^{-1}$ to $45^{\circ}$ and a faint counterclockwise cell 

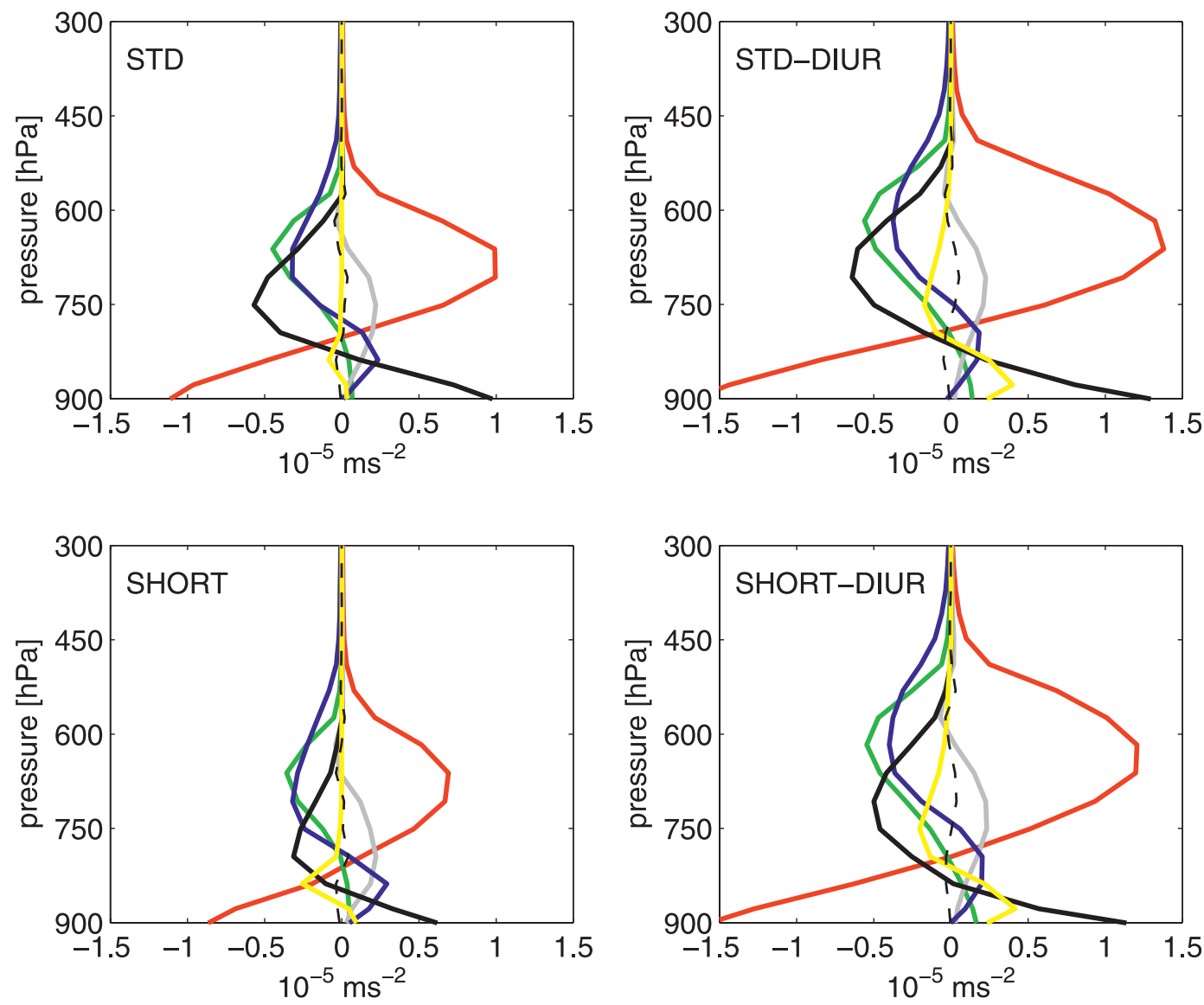

FIG. A2. The zonal momentum balance averaged within $10^{\circ}$ for STD (top left) with disabled diurnal cycle and (top right) with enabled diurnal cycle, and for short-tail stability function (bottom left) with disabled diurnal cycle and (bottom right) with enabled diurnal cycle. Simulation labels are according to Table A1. The color coding is as follows: red-Coriolis force, green - meridional advection of mean relative vorticity, gray-negative of vertical advection of mean zonal momentum, blue-eddy momentum flux convergence (horizontal and vertical eddies), black—vertical diffusion of zonal momentum, yellow—cumulus friction, and black dashed—total sum.

above. The Hadley cell strengthens by $50 \%$ compared to STD when the diurnal cycle is included (Table A1). For daily-averaged insolation, using the short-tail stability function results in a weakening of the Hadley cell strength by $15 \%$ compared to STD. In all simulations, vertical diffusion of meridional momentum decelerates the Hadley cell by downgradient momentum mixing between upper and lower branches as in STD, although at somewhat decreased magnitude (not shown). For the zonal momentum balance, including the diurnal cycle (STD-DIUR) lowers the time-mean depth of the tropical PBL but does not diminish vertical diffusion of zonal momentum in the upper Hadley cell branch (Fig. A2). While vertical diffusion of zonal momentum is reduced near the top of the upper Hadley cell branch when the short-tail stability function is used (SHORT, SHORTDIUR), it remains important in the lower part of the upper branch. Therefore, there is no indication that the strong vertical diffusion of momentum in the upper branch of the snowball Earth Hadley cell is an artifact of using daily-averaged insolation or a long-tail stability function in the parameterization of eddy viscosity.

\section{REFERENCES}

Becker, E., G. Schmitz, and R. Gepraegs, 1997: The feedback of midlatitude waves onto the Hadley cell in a simple general circulation model. Tellus, 49A, 182-199.

Brinkop, S., and E. Roeckner, 1995: Sensitivity of a general circulation model to parameterizations of cloud-turbulence interactions in the atmospheric boundary layer. Tellus, 47A, 197-220.

Caballero, R., R. T. Pierrehumbert, and J. L. Mitchell, 2008: Axisymmetric, nearly inviscid circulations in non-condensing radiative-convective atmospheres. Quart. J. Roy. Meteor. Soc., 134, 1269-1285, doi:10.1002/qj.271.

Frierson, D. M. W., I. M. Held, and P. Zurita-Gotor, 2006: A grayradiation aquaplanet moist GCM. Part I: Static stability and eddy scale. J. Atmos. Sci., 63, 2548-2566. 
Garratt, J. R., 1992: The Atmospheric Boundary Layer. Cambridge University Press, $316 \mathrm{pp}$.

Haberle, R. M., J. B. Pollack, J. R. Barnes, R. W. Zurek, C. B. Leovy, J. R. Murphy, H. Lee, and J. Schaeffer, 1993: Mars atmospheric dynamics as simulated by the NASA Ames general circulation model. 1. The zonal-mean circulation. J. Geophys. Res., 98 (E2), 3093-3123.

Haqq-Misra, J., S. Lee, and D. M. W. Frierson, 2011: Tropopause structure and the role of eddies. J. Atmos. Sci., 68, 2930-2944.

Held, I. M., and A. Y. Hou, 1980: Nonlinear axially symmetric circulations in a nearly inviscid atmosphere. J. Atmos. Sci., 37, 515-533.

_- and B. J. Hoskins, 1985: Large-scale eddies and the general circulation of the troposphere. Advances in Geophysics, Vol. 28A, Academic Press, 3-31, doi:10.1016/ S0065-2687(08)60218-6.

Hoffman, P. F., and D. P. Schrag, 2002: The snowball Earth hypothesis: Testing the limits of global change. Terra Nova, 14, 129-155, doi:10.1046/j.1365-3121.2002.00408.x.

_ - A. J. Kaufman, G. P. Halverson, and D. P. Schrag, 1998: A Neoproterozoic snowball earth. Science, 281, 1342-1346, doi:10. 1126/science.281.5381.1342.

Holtslag, A. A. M., and B. A. Boville, 1993: Local versus nonlocal boundary-layer diffusion in a global climate model. J. Climate, 6, 1825-1842.

Hou, A., 1984: Axisymmetric circulations forced by heat and momentum sources: A simple model applicable to the Venus atmosphere. J. Atmos. Sci., 41, 3437-3455.

Hourdin, F., F. Couvreux, and L. Menut, 2002: Parameterization of the dry convective boundary layer based on a mass flux representation of thermals. J. Atmos. Sci., 59, 1105-1123.

Kim, H.-K., and S. Lee, 2001a: Hadley cell dynamics in a primitive equation model. Part I: Axisymmetric flow. J. Atmos. Sci., 58, $2845-2858$

$\longrightarrow$, and $-2001 \mathrm{~b}$ : Hadley cell dynamics in a primitive equation model. Part II: Nonaxisymmetric flow. J. Atmos. Sci., 58, 2859-2871.

Kirschvink, J. L., 1992: Late Proterozoic low-latitude global glaciation: The snowball Earth. The Proterozoic Biosphere, J. W. Schopf and C. Klein, Eds., Cambridge University Press, 51-52.

Korty, R. L., and T. Schneider, 2008: Extent of Hadley circulations in dry atmospheres. Geophys. Res. Lett., 35, L23803, doi:10.1029/ 2008 GL035847.

Lorenz, E. N., 1967: The Nature and Theory of the General Circulation of the Atmosphere. World Meteorological Organization, $161 \mathrm{pp}$.

Louis, J.-F., 1979: A parametric model of vertical eddy fluxes in the atmosphere. Bound.-Layer Meteor., 17, 187-202.

Medeiros, B., A. Hall, and B. Stevens, 2005: What controls the mean depth of the PBL? J. Climate, 18, 3157-3172.

Mlawer, E. J., S. J. Taubman, P. D. Brown, M. J. Iacono, and S. A. Clough, 1997: Radiative transfer for inhomogeneous atmospheres: RRTM, a validated correlated- $k$ model for the longwave. J. Geophys. Res., 102 (D14), 16 663-16 682.

Nolan, D. S., C. Zhang, and S.-H. Chen, 2007: Dynamics of the shallow meridional circulation around intertropical convergence zones. J. Atmos. Sci., 64, 2262-2285.

Nordeng, T. E., 1994: Extended versions of the convective parametrization scheme at ECMWF and their impact on the mean and transient activity of the model in the tropics. ECMWF Tech. Memo. 206, 41 pp.
Peixoto, J. P., and A. H. Oort, 1992: Physics of Climate. American Institute of Physics, 520 pp.

Pierrehumbert, R. T., 2010: Principles of Planetary Climate. Cambridge University Press, $652 \mathrm{pp}$.

- D. Abbot, A. Voigt, and D. Koll, 2011: Climate of the Neoproterozoic. Annu. Rev. Earth Planet. Sci., 39, 417-460, doi:10.1146/annurev-earth-040809-152447.

Randall, D. A., J. A. Abeles, and T. G. Corsetti, 1985: Seasonal simulations of the planetary boundary layer and boundarylayer stratocumulus clouds with a general circulation model. J. Atmos. Sci., 42, 641-676.

Roeckner, E., and Coauthors, 2003: The atmospheric general circulation model ECHAM5. Part I: Model description. MPI Tech. Rep. 349, Max Planck Institute for Meteorology, $127 \mathrm{pp}$.

Satoh, M., M. Shiobara, and M. Takahashi, 1995: Hadley circulations and their roles in the global angular momentum budget in two- and three-dimensional models. Tellus, 47A, 548-560.

Savijarvi, H., 2009: Stable boundary layer: Parametrizations for local and larger scales. Quart. J. Roy. Meteor. Soc., 135, 914-921.

Schneider, E. K., 1977: Axially symmetric steady-state models of the basic state for instability and climate studies. Part II: Nonlinear calculations. J. Atmos. Sci., 34, 280-296.

Schneider, T., 2006: The general circulation of the atmosphere. Annu. Rev. Earth Planet. Sci., 34, 655-688.

— , and C. C. Walker, 2006: Self-organization of atmospheric macroturbulence into critical states of weak nonlinear eddyeddy interactions. J. Atmos. Sci., 63, 1569-1586.

— the seasonal cycle of a Hadley circulation and implications for monsoon dynamics. J. Atmos. Sci., 65, 915-934.

Soares, P. M. M., P. M. A. Miranda, A. P. Siebesma, and J. Teixeira, 2004: An eddy-diffusivity/mass-flux parametrization for dry and shallow cumulus convection. Quart. J. Roy. Meteor. Soc., 130, 3365-3383, doi:10.1256/qj.03.223.

Stull, R. B., 1988: An Introduction to Boundary Layer Meteorology. Kluwer Academic, 666 pp.

Tiedtke, M., 1989: A comprehensive mass flux scheme for cumulus parameterization in large-scale models. Mon. Wea. Rev., 117, 1779-1800.

Walker, C. C., and T. Schneider, 2005: Response of idealized Hadley circulations to seasonally varying heating. Geophys. Res. Lett., 32, L06813, doi:10.1029/2004GL022304

— ulations with an idealized GCM. J. Atmos. Sci., 63, 3333-3350.

Williams, G. P., 1988a: The dynamical range of global circulations-I. Climate Dyn., 2, 205-260.

_ 1988b: The dynamical range of global circulations-II. Climate Dyn., 3, 45-84.

Wilson, R. J., 1997: A general circulation model simulation of the Martian polar warming. Geophys. Res. Lett., 24, 123-126.

Zhang, C., M. McGauley, and N. A. Bond, 2004: Shallow meridional circulation in the tropical eastern Pacific. J. Climate, 17, 133-139.

— D. S. Nolan, C. D. Thorncroft, and H. Nguyen, 2008: Shallow meridional circulations in the tropical atmosphere. J. Climate, 21, 3453-3470.

Zilitinkevich, S. S., V. L. Perov, and J. C. King, 2002: Near-surface turbulent fluxes in stable stratification: Calculation techniques for use in general-circulation models. Quart. J. Roy. Meteor. Soc., 128, 1571-1587. 